\title{
Comparison of serum adiponectin and osteopontin levels along with metabolic risk factors between obese and lean women with and without PCOS
}

\author{
Suleyman Erkan Alatas', Sevilay Yavuz Dogu², Derya Kilic ${ }^{1}$, Tolga Guler ${ }^{1}$ \\ ${ }^{1}$ Department of Obstetrics and Gynaecology, Pamukkale University Medical School, Denizli, Turkey \\ ${ }^{2}$ Department of Obstetrics and Gynaecology, Istanbul Haseki Research and Education Hospital, Istanbul, Turkey
}

\begin{abstract}
Introduction: The objective of this study was to investigate the possible relation between serum adiponectin and osteopontin levels as metabolic risk markers among women with different polycystic ovary syndrome (PCOS) phenotypes.

Material and methods: In a University Hospital setting PCOS patients diagnosed according to Rotterdam Consensus Conference criteria with body mass index (BMI) between 18 and 35 were recruited.

Results: Overall, 57 PCOS patients and 57 age- and BMI-matched healthy controls were included in the study. Luteinising hormone (LH) to follicle-stimulating hormone FSH ratio (LH/FSH), free androgen index (FAI), and dehydroepiandrosterone sulphate (DHEAS-S) was found to be significantly higher in women with PCOS. There was significant interaction between PCOS status and obesity for serum adiponectin levels. Although mean adiponectin and osteopontin levels were similar among cases and controls, a further two-way ANOVA comparison within lean and obese subgroups revealed adiponectin to be significantly lower in lean PCOS women than in lean controls. LH/FSH ratio and adiponectin levels were all found to differ between lean counterparts; however, they did not show any correlation with metabolic markers [cholesterol, homeostatic model assessment (HOMA) or C-reactive protein (CRP) levels] in overall lean women or in the lean PCOS subgroup.

Conclusion: Serum adiponectin levels in lean PCOS women were significantly lower than those in lean controls. On the other hand, mean adiponectin and osteopontin levels were similar in PCOS cases and controls overall. (Endokrynol Pol 2020; 71 (6): 497-503)

Key words: adiponectin; osteopontin; polycystic ovary syndrome
\end{abstract}

\section{Introduction}

Polycystic ovary syndrome (PCOS) is the most common endocrine disorder, which is characterised by ovulatory abnormalities, hyperandrogenism, and insulin resistance (IR) [1]. According to the Rotterdam criteria, diagnosis of PCOS requires at least two of the following three findings: hyperandrogenism (HA), ovulatory dysfunction (OD), and polycystic ovarian morphology (PCO) [1]. Therefore, the possible combination of these factors results in four different phenotypes $(\mathrm{HA}+\mathrm{OD}, \mathrm{HA}+\mathrm{PCO}, \mathrm{OD}+\mathrm{PCO}$, and $\mathrm{HA}+\mathrm{OD}+\mathrm{PCO})$ all of which are defined as the same disease. Polycystic ovary syndrome represents a heterogeneous genetic condition of reproductive age with a relatively high prevalence of up to $15 \%$ of all women [2]. The exact underlying disease remains still unclear; however, it has been well established that it is not only often associated with ovulatory dysfunction, but also a crucial inflammatory-based metabolic course interrelated with IR plays a crucial role for this syndrome. In line with this fact, IR is detected in $40-70 \%$ of women diagnosed with PCOS [3].

Many putative molecules have been proposed to be involved in PCOS pathogenesis and related metabolic disturbances. Hepatokines, adipokines, myokines, and cytokines that have been reported to be associated with PCOS include, but are not limited to, Fetuin-A, lipocalin-2, adropin, leptin, and irisin [4-7]. It has long been known that women with PCOS appear to have a high risk for central obesity [8]. It was previously established that obesity causes hormonal, metabolic, and inflammatory alterations by means of effecting cytokine, adipokine, and inflammatory mediator secretions [9, 10]. Although obesity accompanies insulin resistance in many cases, non-obese PCOS patients can also present with insulin resistance that can be regarded to be related with inflammation [11]. There is still limited evidence on whether metabolic changes are related to the disease itself or only the results of obesity.

Adipokines are cytokines that are produced mainly by adipose tissue itself and play a key role in insulin 
sensitivity as well as inflammation. It has been documented that adiponectin is actively involved in glucose homeostasis and insulin metabolism [12]. Furthermore, adiponectin is regarded as an insulin sensitising agent because a low level of adiponectin was found to be associated with IR and increased risk of type 2 diabetes mellitus [13]. Interestingly, adiponectin has also been reported to exert anti-inflammatory and antiatherogenic properties [14]. The effects of adiponectin are mediated mainly via two receptors, AdipoR1 and AdipoR2, which are also expressed in female reproductive tissue, such as ovary, placenta, endometrium, and oviduct $[15,16]$. It has been suggested that hypoadiponectinaemia may also play a role in the pathogenesis of PCOS in relation with hyperandrogenaemia, obesity, and IR [16, 17].

Results regarding the role of adiponectin in PCOS have been inconsistent, and it is still unresolved whether adiponectin alteration is intrinsic to PCOS pathophysiology or secondary to PCOS-associated obesity and other metabolic alterations [18]. In addition, there is strong evidence that IR is related with adipose tissue involving the secretion of inflammatory cytokines [19]. Immune modulator molecules may play a role in inflammatory pathways in several ways: Firstly, they can promote cell recruitment to inflammatory sites; secondly, they may effect cell activation and cytokine secretion; and lastly, they can regulate cell cycle and apoptosis of several cell types. Osteopontin (OPN) is a multi-functional extracellular matrix-associated phosphoprotein that plays a remarkable role in cell macrophage activation, chemotaxis, vascularisation, inflammation, matrix remodelling, and inhibition of apoptotic activities $[19,20]$. It has been proposed that osteopontin plays an important role in macrophage recruitment to inflammation in adipose tissue and contributes to chronic low-grade inflammation [20]. In relation to macrophage infiltration and inflammation, OPN is also demonstrated to associate in the pathogenesis of atherosclerotic process, insulin resistance, and malignant disorders [19]. Recently only a few studies have indicated that increased osteopontin levels may be associated with PCOS in selected populations [14, 21].

To date, many molecular markers have been investigated in PCOS patients; however, no exact cause and effect could be documented except significant associations. The main reason for this is the fact that the pathogenesis of PCOS is complex, phenotypes are heterogenous, and the disease is not limited to obese women. Heterogeneity is an inherent feature of PCOS from its aetiology to its presentation [4]. PCOS presentation is not homogenous, and in terms of manifestation, patients can also be grouped in to different types such as metabolic PCOS or reproductive PCOS [22]. Ozga et al. argued that metabolic and androgenic status of different PCOS phenotypes may differ. More data are needed to define the long-term prognosis and monitor complications associated with these alterations in different PCOS phenotypes. Therefore, investigating the clinical usefulness of putative markers holds importance not only for dissecting the pathophysiology of the disease, but also for understanding the association of these markers with further complications. Because adiponectin and osteopontin are reported, as putative markers, to play a role in the pathogenesis and clinical manifestation of PCOS, it would be important to evaluate them to define both their individual roles and their association with each other. To our knowledge, there has been no report to analyse the association of both in the same population according to body mass index (BMI). Therefore, this study aimed to investigate the possible relation between serum osteopontin and adiponectin levels as metabolic risk markers among women with different PCOS phenotypes. We also intended to evaluate the correlation of metabolic profiles in distinct PCOS phenotypes with respect to metabolic disturbances.

\section{Material and methods}

\section{Study population}

Female patients between the ages of 18 and 45 years, who were diagnosed according to Rotterdam Consensus Conference criteria, were included in the study. The control group consisted of agematched healthy women who attended the gynaecology clinic for contraception or other gynaecological issues. Matching was achieved in lean and obese counterparts by recruiting one control who was within five years of age of the patient. This study was conducted in a University Hospital setting. Institutional Ethical Board approval was obtained for the study, and all participants gave consent.

Inclusion criteria were defined as follows: $18-45$ years old PCOS patients diagnosed according to Rotterdam Consensus Conference criteria, exclusion of other endocrinopathies, having spontaneous menarche and normal sexual development, and BMI between 18 and 35. Exclusion criteria were defined as follows: taking medications that can affect sex steroid levels and menstruation, previous diagnosis of diabetes mellitus and taking antidiabetic drugs, chronic renal or liver disease, haematological disorders, history of any oncological disease, and chemo/radiotherapy. Women incidentally diagnosed with elevated liver tests were also excluded because their condition might have affect serum hormone levels and the exact cause of this elevation was not established at the time of serum collection and analysis. According to the inclusion and exclusion criteria, 57 women were included in the PCOS group for comparison with age- and BMI-matched healthy controls. Institutional Ethical Board approval was obtained for the study, and all participants gave consent.

\section{Evaluation and measurements}

Menstrual and gynaecological history was assessed by a gynaecologist using a standardised investigation form. Irregular cycles were defined as follows: oligomenorrhoea, cycle length between 36 and 180 days; or amenorrhoea, absence of any menstrual bleeding for more than 180 days. Either Modified Ferriman-Gallway (F-G) scores (evaluated at least two months after any kind of epilation) of more than 8 or the presence of acne and/or alopecia was regarded as clinical HA. Biochemical hyperandrogenism was defined when 
total testosterone, free testosterone, dehydroepiandrosterone sulphate and/or androstenedione exceeded laboratory limits or when calculated free androgen index (FAI) was greater than 5 . Anthropometric measurements of the women were noted, and the BMI and waist-to-hip ratio (WHR) were calculated. Blood samples were collected during the early follicular phase after overnight fasting. Follicle-stimulating hormone (FSH), luteinising hormone (LH), oestradiol (E2), progesterone (P), 17-hydroxyprogesterone (17-OHP), prolactin, sex hormone-binding globulin (SHBG), total testosterone (T), dehydroepiandrosterone sulphate (DHEAS), total cholesterol, low-density lipoprotein (LDL), high-density lipoprotein (HDL), very low-density lipoprotein (VLDL), triglyceride, complete blood count, renal and liver function markers, and total urine analysis were assayed. Free androgen index (FAI) was calculated according to the defined formula:

\section{$(T \times 100) / S H B G$}

Insulin resistance was also calculated using the following formula:

(fasting glucose $[\mathrm{mg} / \mathrm{dL}] \times$ fasting insulin [uIU])/405

and recorded as HOMA-IR. Serum adiponectin and osteopontin levels were determined by sandwich ELISA using secondary rabbit antibodies as described by the manufacturer (B-Bridge International, Inc., USA). Total testosterone, SHBG, DHEAS, TSH, and prolactin concentrations were measured by electrochemiluminescent immunoassay with a inter- and intra-assay coefficient of variation $(\mathrm{CV})$ of $<5 \%$. The serum levels of androstenedione and free testosterone were analysed using the radioactive immunoassay method. Total cholesterol, HDL, and triglycerides were analysed using an enzymatic colorimetric assay. Analyses were performed on Cobas e602 apparatus (Roche Diagnostics GmbH, Manheim, Germany). Pelvic or transvaginal sonographic (when suitable) evaluation was performed on the same day of blood sample collection.

\section{Statistical analysis}

The sample size was calculated in order to achieve a power of $95 \%$ with an alpha error of 0.05 . The minimum sample size in each groups of lean and obese women was calculated by G-power to be at least 28 for serum osteopontin comparison with an effect size of 0.985 according to reported values of $69.12 \pm 31.59 \mathrm{ng} / \mathrm{mL} v s .42 .66$ $\pm 21.28 \mathrm{ng} / \mathrm{mL}$ by Saklamaz et al. [14]. In total 28 and 29 women were included to these groups that added up to 57 cases and 57 controls with a one-to-one case-control ratio. This sample size was also suitable to achieve the same power for serum adiponectin comparisons with an effect size of 3.385 according to the results of Baldani et al. [18].

Statistical tests were performed by PSPP 1.0.1 and R software using EasyR plugin. Numerical variables were presented as mean \pm standard deviation (SD). Categorical parameters were presented along with their percentages. Normality tests were performed by distribution analysis and Kolmogorov-Smirnov test. Parametric Student's t-test was used when normality was reached; otherwise, Mann-Whitney $\mathrm{U}$ test was utilised. $\mathrm{p}<.05$ was considered as statistically significant.

\section{Results}

Overall, 57 PCOS patients and 57 age- and BMI-matched healthy controls were included to the study. Patients with a BMI between 18 and 35 were selected for analysis in order to eliminate outlier values. Patients and cases were further divided into lean and obese groups according to BMI below or above of 25. Altogether, there were total 28, 29, 28, and 29 lean control, obese control, lean PCOS, and obese PCOS participants, respectively. Mean age, BMI, and WHR among control and PCOS groups were similar. The baseline characteristics of the two main groups along with four subgroups are presented in Table 1.

The laboratory parameters of the PCOS and the controls were investigated and are presented in Table 2, including all subgroups. Among the investigated laboratory parameters $\mathrm{LF} / \mathrm{FSH}$ ration, FAI, and dehydroepiandrosterone sulphate (DHEAS-S) were found to be significantly different between the PCOS and control groups. A post-hoc analysis among lean and obese counterparts, however, revealed that FAI differed only between obese control and obese PCOS women but not in lean cases and controls. The only parameter that significantly differed between lean controls and lean PCOS women appeared to be the $\mathrm{LH} / \mathrm{FSH}$ ratio. On the other hand, LH/FSH and FAI were significantly different between obese control and obese PCOS cases. DHEA$S$ was not found to significantly differ between lean control and lean PCOS or between obese control and obese PCOS women. For the PCOS cases, being lean or obese significantly affected total cholesterol, LDL, HDL, and C-reactive protein (CRP) levels.

Mean adiponectin levels were investigated by two-way ANOVA. This analysis revealed slightly signifi-

Table 1. Anthropometric and sonographic characteristics of the participants

\begin{tabular}{|c|c|c|c|c|c|c|c|}
\hline Parameters & $\begin{array}{c}\text { Control Group } \\
\text { Overall } \\
\begin{array}{c}(\mathrm{n}=57) \\
X \pm S D\end{array}\end{array}$ & $\begin{array}{c}\text { PCOS Group } \\
\text { Overall } \\
(\mathrm{n}=57) \\
X \pm S D\end{array}$ & $\mathbf{p}^{*}$ & $\begin{array}{l}\text { Lean Control } \\
\qquad \begin{array}{l}(\mathrm{n}=28) \\
X \pm S D\end{array}\end{array}$ & $\begin{array}{l}\text { Obese Control } \\
\begin{array}{c}(\mathrm{n}=29) \\
\mathrm{X} \pm S D\end{array}\end{array}$ & $\begin{array}{c}\text { Lean PCOS } \\
(\mathrm{n}=28) \\
X \pm S D\end{array}$ & $\begin{array}{c}\text { Obese PCOS } \\
\begin{array}{c}(n=29) \\
X \pm S D\end{array}\end{array}$ \\
\hline Age [yrs] & $27.2 \pm 8.3$ & $25.0 \pm 5.8$ & 0.1 & $22.4 \pm 5.0$ & $31.8 \pm 8.3$ & $22.6 \pm 5.0$ & $27.2 \pm 5.7$ \\
\hline BMI $\left[\mathrm{kg} / \mathrm{m}^{2}\right]$ & $25.0 \pm 5.1$ & $24.6 \pm 4.5$ & 0.7 & $20.9 \pm 1.9$ & $28.8 \pm 4.2$ & $20.6 \pm 1.3$ & $28.4 \pm 3.0$ \\
\hline WHR & $0.76 \pm 0.06$ & $0.78 \pm 0.07$ & 0.3 & $0.74 \pm 0.05$ & $0.79 \pm 0.05$ & $0.74 \pm 0.05$ & $0.81 \pm 0.06$ \\
\hline Antral follicle count & $4.4 \pm 2.9$ & $16.7 \pm 4.4$ & $<0.001$ & $4.4 \pm 2.8$ & $4.4 \pm 2.9$ & $17.0 \pm 4.7$ & $16.5 \pm 4.1$ \\
\hline Ovarian volume $\left[\mathrm{cm}^{3}\right]$ & $8.7 \pm 2.3$ & $11.9 \pm 2.9$ & $<0.001$ & $8.6 \pm 2.0$ & $8.8 \pm 2.5$ & $12.4 \pm 3.0$ & $11.6 \pm 3.0$ \\
\hline
\end{tabular}

${ }^{*}$ p values were calculated between PCOS and control group; PCOS — polycystic ovarian syndrome; BMI — body mass index; WHR — waist-hip ratio 
Table 2. Laboratory parameters among control, polycystic ovarian syndrome (PCOS) group, and subgroups

\begin{tabular}{|c|c|c|c|c|c|c|c|}
\hline Parameters & $\begin{array}{c}\text { Control Group } \\
\text { Overall } \\
(\mathrm{n}=57) \\
X \pm S D\end{array}$ & $\begin{array}{l}\text { PCOS Group } \\
\text { Overall } \\
\text { (n = 57) } \\
X \pm S D\end{array}$ & $\mathbf{p}^{*}$ & $\begin{array}{l}\text { Lean Control } \\
\begin{array}{l}(\mathrm{n}=28) \\
X \pm S D\end{array}\end{array}$ & $\begin{array}{l}\text { Obese Control } \\
\qquad \begin{array}{c}(n=29) \\
X \pm S D\end{array}\end{array}$ & $\begin{array}{c}\text { Lean PCOS } \\
\begin{array}{c}(\mathrm{n}=28) \\
X \pm S D\end{array}\end{array}$ & $\begin{array}{c}\text { Obese PCOS } \\
\begin{array}{c}(\mathrm{n}=29) \\
X \pm S D\end{array}\end{array}$ \\
\hline LH/FSH ratio & $0.8 \pm 0.4$ & $1.5 \pm 0.9$ & $<0.001$ & $0.9 \pm 0.4$ & $0.7 \pm 0.7$ & $1.5 \pm 1.2$ & $1.5 \pm 1.5$ \\
\hline $\mathrm{FAl}$ & $0.7 \pm 0.6$ & $1.6 \pm 1.9$ & 0.001 & $0.8 \pm 0.7$ & $0.6 \pm 0.4$ & $1.2 \pm 1.3$ & $2.0 \pm 2.3$ \\
\hline SHBG & $46.8 \pm 16.9$ & $45.8 \pm 41.6$ & 0.9 & $47.2 \pm 16.6$ & $46.5 \pm 17.7$ & $54.3 \pm 52.4$ & $37.6 \pm 25.9$ \\
\hline DHEA-S & $220.5 \pm 128.9$ & $268.4 \pm 122.7$ & 0.04 & $256.8 \pm 144.6$ & $185.4 \pm 102.3$ & $277.8 \pm 126.1$ & $259.3 \pm 221.7$ \\
\hline Total cholesterol & $173.4 \pm 29.9$ & $171.1 \pm 36.5$ & 0.7 & $159.8 \pm 28.4$ & $186.5 \pm 25.5$ & $159.6 \pm 36.4$ & $182.3 \pm 33.7$ \\
\hline LDL & $100.6 \pm 27.1$ & $97.0 \pm 29.8$ & 0.5 & $88.1 \pm 23.7$ & $112.7 \pm 24.8$ & $85.1 \pm 25.1$ & $108.4 \pm 29.8$ \\
\hline HDL & $55.2 \pm 12.8$ & $53.9 \pm 13.7$ & 0.6 & $57.8 \pm 13.9$ & $52.7 \pm 11.3$ & $58.7 \pm 13.8$ & $49.2 \pm 12.2$ \\
\hline Triglyceride & $88.9 \pm 42.4$ & $101.7 \pm 55.1$ & 0.2 & $72.2 \pm 30.4$ & $105.1 \pm 46.5$ & $81.0 \pm 28.7$ & $121.5 \pm 66.7$ \\
\hline Fasting glucose & $86.8 \pm 11.2$ & $84.7 \pm 14.1$ & 0.4 & $83.3 \pm 8.4$ & $90.0 \pm 12.7$ & $83.6 \pm 19.3$ & $85.6 \pm 5.8$ \\
\hline HOMA-IR & $2.2 \pm 1.2$ & $2.7 \pm 1.9$ & 0.2 & $1.84 \pm 0.98$ & $2.6 \pm 1.2$ & $2.4 \pm 2.2$ & $2.8 \pm 1.6$ \\
\hline CRP & $0.21 \pm 0.25$ & $0.28 \pm 0.58$ & 0.4 & $0.15 \pm 0.19$ & $0.27 \pm 0.29$ & $0.13 \pm 0.15$ & $0.43 \pm 0.76$ \\
\hline
\end{tabular}

*p values were calculated between PCOS and control group; PCOS — polycystic ovarian syndrome, LH/FSH — luteinising hormone/follicle-stimulating hormone; FAI — free androgen index; SHBG — sex hormone binding globulin; DHEA-S — dehydroepiandrosterone sulphate; LDL — low-density lipoprotein; HDL — high-density lipoprotein; HOMA-IR — homeostatic model assessment of insulin resistance; CRP — C-reactive protein

cant interaction between PCOS status and obesity for serum adiponectin levels, $\mathrm{F}(1,110)=4.567, \mathrm{p}=0.035$, partial eta squared $=.040$. Therefore, we further calculated the simple main effects. Because this interaction seems to be ordinal we also measured the main effect, which was found to be insignificant for adiponectin between PCOS and controls, $\mathrm{F}(1,110)=3.021$, $\mathrm{p}=.085$, partial eta squared $=.027$. For the lean women the mean adiponectin level was $4.2 \mathrm{ng} / \mathrm{mL}$ (95\% CI, 1.1 to 7.2) lower in PCOS cases than controls, $\mathrm{F}(1,110)=7.38$, $\mathrm{p}=.008$, partial eta squared $=.063$ ). However, the simple main effect of PCOS status on adiponectin levels for those being obese was not statistically significant, $\mathrm{F}(1,110)=.081, \mathrm{p}=.776$, partial eta squared $=.001)$. On the other hand, for the mean serum osteopontin levels, there was no statistically significant interaction between PCOS status and obesity, $\mathrm{F}(1,110)=.520$, $\mathrm{p}=.473$, partial eta squared $=.005$. There was a sta- tistically significant main effect of obesity on mean osteopontin levels, $\mathrm{F}(1,110)=79.003, \mathrm{p}<.001$, partial eta squared $=.418$ ) The mean serum osteopontin levels were markedly higher in obese PCOS and obese controls than in lean PCOS and controls (42.1, 44.1, 15.5 , and 12.8, respectively). However, there was no statistically significant difference in osteopontin levels between the PCOS and control groups, $\mathrm{F}(1,110)=.011$, $\mathrm{p}=.918$, partial eta squared $<.001$. A summary of the comparisons of control and PCOS cases after defining subgroups for obesity with respect to adiponectin, osteopontin, LH/FSH, FAI, SHBG, and DHEA-S are shown in Table 3.

Since only LH/FSH and adiponectin levels were found to be significantly different among lean control and lean PCOS patients, these parameters were investigated with respect to metabolic risk factors. Neither overall lean women nor the lean PCOS subgroup

Table 3. Comparison of control and polycystic ovarian syndrome (PCOS) cases with respect to adiponectin, osteopontin, luteinising hormone/follicle-stimulating hormone (LH/FSH), free androgen index (FAI), sex hormone binding globulin (SHBG), and dehydroepiandrosterone sulphate (DHEA-S) after defining subgroups for obesity

\begin{tabular}{lcc}
\hline & Lean control vs. lean PCOS & Obese control vs. obese PCOS \\
\hline LH/FSH ratio & Significant difference & Significant difference \\
\hline FAl & NS & Significant difference \\
\hline SHBG & NS & NS \\
\hline DHEA-S & NS & NS \\
\hline Adiponectin & Significant difference & NS \\
\hline Osteopontin & NS & NS \\
\hline
\end{tabular}

LH/FSH — luteinising hormone/follicle-stimulating hormone; FAI — free androgen index; SHBG — sex hormone binding globulin; DHEA-S — dehydroepiandrosterone sulphate; NS — non-significant 
showed significant correlation with the cholesterol panel, HOMA, or CRP levels.

\section{Discussion}

In this study we compared 57 PCOS patients with 57 age- and BMI-matched healthy controls with respect to their serum adiponectin and osteopontin values. Both groups were also divided into two subgroups: lean and obese. Antral follicle count and ovarian volume were found to be higher in the PCOS group, as expected. Similarly, LF/FSH ration, FAI, and DHEAS-S were found to be significantly higher in women with PCOS. Interestingly the only baseline parameter that significantly differed between lean control and lean PCOS women appeared to be the LH/FSH ratio. Although mean adiponectin and osteopontin levels were similar among cases and controls, a further two-way ANOVA comparison within lean and obese subgroups revealed additional information such as adiponectin being significantly different between lean control and lean PCOS women. The $\mathrm{LH} / \mathrm{FSH}$ ratio and adiponectin levels were found to differ between lean counterparts, but they did not show any correlation with metabolic markers (cholesterol panel, homeostatic model assessment [HOMA] or CRP levels), nor in overall lean women or in the lean PCOS subgroup.

Metabolic dysfunction and chronic low-grade inflammation are the two main pathways involved because they are mainly induced by obesity and hyperandrogenism [23]. Daan et al. investigated the metabolic and inflammatory biomarker profiles in women with PCOS such as adipocytokines: interleukins (IL) - IL-1b, IL-6, IL-13, IL-17, IL-18, tumour necrosis factor alpha (TNF- $\alpha$ ), adiponectin, adipsin, leptin, chemerin, resistin, retinol-binding protein 4 (RBP4), soluble CD26/dipeptidyl peptidase-IV (DPP-IV/sCD26), monocyte chemoattractant protein-1 (CCL2/MCP-1), growth factors - placental growth factor (PIGF), vascular endothelial growth factor (VEGF) and its soluble receptor (sVEGF-R1), and soluble cell adhesion molecules - soluble intercellular adhesion molecule 1 (sICAM-1/sCD54) and soluble vascular cell adhesion molecule 1 (sVCAM-1/sCD106) [24]. Their analysis revealed adiponectin as a potential discriminative marker for PCOS, and it was closely related with BMI. Adiponectin is a cytokine showing anti-inflammatory features and inversely correlating with visceral adiposity and obesity [12]. Deregulations in its secretion have been speculated to be involved in PCOS pathogenesis [25]. Besides this, it may also be possible that PCOS independently affects this adipokine expression. Other studies also demonstrated hypoadiponectinaemia in PCOS patients irrespective of obesity; however, we failed to establish a link between PCOS and serum levels of adiponectin in obese women [26, 27]. Similarly, Baldani et al. did not find a link between abdominal fat distribution and serum adiponectin levels [18]. In contrast, Gandevani et al. reported that serum adiponectin levels were similar between obese and non-obese controls, but they found decreased adiponectin levels in obese PCOS women compared to their non-obese counterparts [28]. In this study, although no significant relation between PCOS and adiponectin levels was found, pairwise comparisons revealed that mean adiponectin levels were affected by there being PCOS only in the lean group. In other words, the significant interaction found in our study for PCOS and obesity showed that having PCOS did have a significant effect for the adiponectin levels in lean but not in obese women. This result is interesting because it indicates the possibility that adiponectin may have a much more associated role in the pathogenesis of PCOS in lean women than in obese women.

The main strength of this study was the comparison of relatively homogenous PCOS group with age and BMI-matched controls. Moreover, HOMA-IR and cholesterol levels between these groups were also comparable. The main reason for this similarity may be the fact that both groups were also stratified for obese and lean counterparts. However, this condition diminishes the generalisability of these results to a larger population in which this kind of matching is not present.

Osteopontin is another cytokine with pro-inflammatory effects that is different from adiponectin. On the other hand, it is similarly related with adipose tissue, and it was previously shown in an animal obesity model that its expression increases in adipose tissue [29]. It was also shown that osteopontin is related with insulin resistance and adipose tissue inflammation [30]. A recent study by Saklamaz et al. documented that osteopontin levels were increased in PCOS [19]. They also documented that serum osteopontin levels in overweight/obese women were higher than in lean women regardless of PCOS. In this study, we similarly found no interaction with PCOS status and obesity for osteopontin levels. However, we did not find any relationship between PCOS status and osteopontin levels in main effect comparison, but we observed a very distinct increment in the overall obese group. Therefore, one explanation for the insignificant relation found in our study may be related with this high correlation of osteopontin with obesity. However, continuation of this insignificancy in the lean counterparts hampers the logic for this comment. Therefore, one can speculate that osteopontin is not involved in PCOS pathogenesis as much as adiponectin. Another speculative explanation would be one or more confounders except for obesity may be related with such 
a finding. Further studies with larger sample sizes and homogeneous subgroups are needed to draw definitive conclusions. It should be noted that the PCOS and control groups in this study had quite similar IR and therefore these results could not be generalised to other populations. Another limitation in this study may arise from age differences between the PCOS and control groups. Although matching for age resulted in no significance of age difference, it should be kept in mind that serum adiponectin levels may be affected by the slightly older age in the control group.

In this study, only the LH/FSH ratio and adiponectin levels were found to significantly differ between lean controls and lean PCOS women. We were, however, unable to document a significant correlation of LH/FSH ratio and adiponectin levels with cholesterol panel, HOMA, or CRP levels. In our results, the correlation between adiponectin and other metabolic risk factors was limited. However, the distinct result we found in the lean subgroup may indicate an important clinical association in the lean PCOS subgroup. One sensational speculation may also be derived from this lack of correlation; adiponectin levels in lean women may stand as a long-term metabolic risk factor, being much more sensitive than lipid profile or HOMA calculation. This possibility should further be investigated prospectively in a larger subgroup.

\section{Conclusion}

Serum adiponectin levels were significantly lower in lean PCOS women compared to lean controls; however, PCOS status did not affect adiponectin levels in obese women. On the other hand, mean adiponectin and osteopontin levels were similar between PCOS cases and controls overall. There is a need for prospective research investigating the clinical value of adiponectin as a long-term metabolic risk factor in lean cases.

\section{Conflict of interests}

The authors state that they have no conflict of interests.

\section{Acknowledgements}

None.

\section{References}

1. Rotterdam ESHRE/ASRM-Sponsored PCOS Consensus Workshop Group. Revised 2003 consensus on diagnostic criteria and long-term health risks related to polycystic ovary syndrome (PCOS). Hum Reprod. 2004; 19(1): 41-47, doi: 10.1093/humrep/deh098, indexed in Pubmed: 14688154.

2. March WA, Moore VM, Willson KJ, et al. The prevalence of polycystic ovary syndrome in a community sample assessed under contrasting diagnostic criteria. Hum Reprod. 2010; 25(2): 544-551, doi: 10.1093/humrep/dep399, indexed in Pubmed: 19910321.

3. Diamanti-Kandarakis E, Dunaif A. Insulin resistance and the polycystic ovary syndrome revisited: an update on mechanisms and implica- tions. Endocr Rev. 2012; 33(6): 981-1030, doi: 10.1210/er.2011-1034, indexed in Pubmed: 23065822

4. Liu S, Hu W, He Y, et al. Serum Fetuin-A levels are increased and associated with insulin resistance in women with polycystic ovary syndrome. BMC Endocr Disord. 2020; 20(1): 67, doi: 10.1186/s12902-020-0538-1, indexed in Pubmed: 32429902.

5. Yilmaz Ö, Temur M, Calan M, et al. The relationship between lipocalin-2 and free testosterone levels in polycystic ovary syndrome. Endokrynol Pol. 2017; 68(1): 7-12, doi: 10.5603/EP.2017.0002, indexed in Pubmed: 28255975.

6. Kuliczkowska-Płaksej J, Mierzwicka A, Jończyk M, et al. Preptin in women with polycystic ovary syndrome. Gynecol Endocrinol. 2018; 34(6): 470-475, doi: 10.1080/09513590.2017.1409715, indexed in Pubmed: 29374985.

7. Bostancı MS, Akdemir N, Cinemre B, et al. Serum irisin levels in patients with polycystic ovary syndrome. Eur Rev Med Pharmacol Sci. 2015; 19(23): 4462-4468, indexed in Pubmed: 26698239.

8. Lim SS, Davies MJ, Norman RJ, et al. Overweight, obesity and central obesity in women with polycystic ovary syndrome: a systematic review and meta-analysis. Hum Reprod Update. 2012; 18(6): 618-637, doi: 10.1093/humupd/dms030, indexed in Pubmed: 22767467.

9. Spritzer PM, Lecke SB, Satler F, et al. Adipose tissue dysfunction, adipokines, and low-grade chronic inflammation in polycystic ovary syndrome. Reproduction. 2015; 149(5): R219-R227, doi: 10.1530/REP-14-0435, indexed in Pubmed: 25628442

10. Rimmer M, Tan BK, Teede H, et al. Metabolic inflexibility in women with polycystic ovary syndrome: a systematic review. Gynecol Endocrinol. 2020; 36(6): 501-507, doi: 10.1080/09513590.2019.1698025, indexed in Pubmed: 31793357.

11. Mehta NN, McGillicuddy FC, Anderson PD, et al. Experimental endotoxemia induces adipose inflammation and insulin resistance in humans. Diabetes. 2010; 59(1): 172-181, doi: 10.2337/db09-0367, indexed in Pubmed: 19794059

12. Rolland YM, Haren MT, Patrick P, et al. Adiponectin levels in obese and non-obese middle-aged African-American women. Obes Res Clin Pract. 2007; 1(1): 1-78, doi: 10.1016/j.orcp.2006.10.002, indexed in Pubmed: 24351429.

13. Yadav A, Kataria MA, Saini V, et al. Role of leptin and adiponectin in insulin resistance. Clin Chim Acta. 2013; 417: 80-84, doi: 10.1016/j. cca.2012.12.007, indexed in Pubmed: 23266767.

14. Ouchi N, Walsh K. Adiponectin as an anti-inflammatory factor. Clin Chim Acta. 2007; 380(1-2): 24-30, doi: 10.1016/j.cca.2007.01.026, indexed in Pubmed: 17343838.

15. Toulis KA, Goulis DG, Farmakiotis D, et al. Adiponectin levels in women with polycystic ovary syndrome: a systematic review and a meta-analysis. Hum Reprod Update. 2009; 15(3): 297-307, doi: 10.1093/humupd/dmp006, indexed in Pubmed: 19261627.

16. Singh A, Bora P, Krishna A. Direct action of adiponectin ameliorates increased androgen synthesis and reduces insulin receptor expression in the polycystic ovary. Biochem Biophys Res Commun. 2017; 488(3): 509-515, doi: 10.1016/j.bbrc.2017.05.076, indexed in Pubmed: 28522293.

17. Ożegowska K, Bartkowiak-Wieczorek J, Bogacz A, et al. Relationship between adipocytokines and angiotensin converting enzyme gene insertion/deletion polymorphism in lean women with and without polycystic ovary syndrome. Gynecol Endocrinol. 2020; 36(6): 496-500, doi: 10.108 0/09513590.2019.1695248, indexed in Pubmed: 31814467.

18. Baldani DP, Skrgatic L, Kasum M, et al. Altered leptin, adiponectin, resistin and ghrelin secretion may represent an intrinsic polycystic ovary syndrome abnormality. Gynecol Endocrinol. 2019; 35(5): 401-405, doi: 10.1080/09513590.2018.1534096, indexed in Pubmed: 30623695.

19. Saklamaz A, Calan M, Yilmaz O, et al. Polycystic ovary syndrome is associated with increased osteopontin levels. Eur J Endocrinol. 2016; 174(4): 415-423, doi: 10.1530/EJE-15-1074, indexed in Pubmed: 26701868.

20. Kuwabara Y, Katayama A, Tomiyama R, et al. Gonadotropin regulation and role of ovarian osteopontin in the periovulatory period. J Endocrinol. 2015; 224(1): 49-59, doi: 10.1530/JOE-14-0203, indexed in Pubmed: 25352284

21. Wang Y, Zhou W, Wu C, et al. Circulating osteopontin and its association with liver fat content in non-obese women with polycystic ovary syndrome: a case control study. Reprod Biol Endocrinol. 2018; 16(1): 31, doi: 10.1186/s12958-018-0331-4, indexed in Pubmed: 29587769.

22. Ożga K, Krzyczkowska-Sendrakowska M, Hubalewska-Dydejczyk A, et al. The value of the free androgen index depends on the phenotype of polycystic ovary syndrome - a single-centre experience. Endokrynol Pol. 2019; 70(4): 330-335, doi: 10.5603/EP.a2019.0017, indexed in Pubmed: 30938834

23. Lim SS, Davies MJ, Norman RJ, et al. Overweight, obesity and central obesity in women with polycystic ovary syndrome: a systematic review and meta-analysis. Hum Reprod Update. 2012; 18(6): 618-637, doi: 10.1093/humupd/dms030, indexed in Pubmed: 22767467.

24. Daan NMP, Koster MPH, de Wilde MA, et al. Biomarker Profiles in Women with PCOS and PCOS Offspring; A Pilot Study. PLoS One. 
2016; 11(11): e0165033, doi: 10.1371/journal.pone.0165033, indexed in Pubmed: 27806063.

25. Polak K, Czyzyk A, Simoncini T, et al. New markers of insulin resistance in polycystic ovary syndrome. J Endocrinol Invest. 2016; 40(1): 1-8, doi: 10.1007/s40618-016-0523-8, indexed in Pubmed: 27473078.

26. Escobar-Morreale HF, Villuendas G, Botella-Carretero JI, et al. Adiponectin and resistin in PCOS: a clinical, biochemical and molecular genetic study. Hum Reprod. 2006; 21(9): 2257-2265, doi: 10.1093/humrep/del146, indexed in Pubmed: 16675483.

27. Sieminska L, Marek B, Kos-Kudla B, et al. Serum adiponectin in women with polycystic ovarian syndrome and its relation to clinical, metabolic and endocrine parameters. J Endocrinol Invest. 2004; 27(6): 528-534, doi: 10.1007/BF03347474, indexed in Pubmed: 15717649.
28. Behboudi-Gandevani S, Ramezani Tehrani F, Bidhendi Yarandi R, et al. The association between polycystic ovary syndrome, obesity, and the serum concentration of adipokines. J Endocrinol Invest. 2017; 40(8): 859-866, doi: 10.1007/s40618-017-0650-x, indexed in Pubmed: 28332170

29. Kiefer FW, Zeyda M, Todoric J, et al. Osteopontin expression in human and murine obesity: extensive local up-regulation in adipose tissue but minimal systemic alterations. Endocrinology. 2008; 149(3): 1350-1357, doi: 10.1210/en.2007-1312, indexed in Pubmed: 18048491.

30. Nomiyama T, Perez-Tilve D, Ogawa D, et al. Osteopontin mediates obesity-induced adipose tissue macrophage infiltration and insulin resistance in mice. J Clin Invest. 2007; 117(10): 2877-2888, doi: 10.1172/JCI31986, indexed in Pubmed: 17823662 